HAIBO WANG, Ph.D. ${ }^{1}$

E-mail: sdlgwanghaibo@sohu.com

HAIQING SI, Ph.D. ${ }^{1}$

(Corresponding author)

E-mail: sihaiqing@126.com

XIAOYUAN WANG, Ph.D. ${ }^{2}$

E-mail:sql370@163.com

${ }^{1}$ School of Civil Aviation and Flight

Nanjing University of Aeronautics and Astronautics

Nanjing 211106, China

2 College of Electromechanical Engineering

Qingdao University of Science and Technology

Qingdao 266000, China
Human-Transport Interaction

Original Scientific Paper

Submitted: 25 Feb. 2018

Accepted: 5 Feb. 2019

\title{
CYCLIST'S INTENTION IDENTIFICATION ON PEDESTRIAN-BICYCLE MIXED SECTIONS BASED ON PHASE-FIELD COUPLING THEORY
}

\begin{abstract}
Bicycle is one of the main factors that affects the traffic safety and capacity on pedestrian-bicycle mixed traffic sections. It is important for implementing the warning of bicycle safety and improving the active safety to identify the cyclists' intention in the mixed traffic environments under the condition of the "Internet of Things". The phase-field coupling theory has been developed in this paper to comprehensively analyse the generation, spring up, increase, transfer, regression and reduction method of the traffic phase. The adaptive genetic algorithm based on the information entropy has been used to extract feature vectors of different types of cyclists for intention identification from the reduced pedestrian-bicycle traffic phase, and the theory of evidence has been provided here to build the identification model. The experimental verification shows that the extraction method of cyclists' intention feature vector and identification model are scientific and reasonable. The theoretical basis can be applied to establishing the pedestrian-bicycle interactive security system.
\end{abstract}

\section{KEY WORDS}

phase-field coupling theory; pedestrian-bicycle mixed traffic section; intention identification; safety warning; traffic phase;

\section{INTRODUCTION}

With the rapid development of automotive industry and the improvement of people's living standards, more and more people have cars. As a result, the motorways in many cities are being constantly broadened, the lane of non-motorized traffic for pedestrians and bicycles is being gradually narrowed, and the conflict between bicycles and pedestrians is becoming more and more serious. The extensive use of the mobile sensor devices (Smartphone, etc.) equipped with GPS and the vigorous development of the "Internet of Things" can provide a powerful guarantee for the study of the improvement of active safety through timely warning and the synergy between pedestrians and bicycles. How to predictably and dynamically identify the cyclist's intention in the pedestrian-bicycle mixed environment is the core scientific issue which needs to be solved urgently.

Domestic and foreign scholars have done a lot of studies on bicycle traffic. At the macro level, the research mainly includes bicycle group behaviour and the relationship of three parameters (traffic flow, traffic density, traffic speed) for bicycle traffic flow model. At the micro level, bicycle traffic was mainly studied using the car-following model, cellular automata model and simulation model based on two-dimensional space. According to the car-following behaviour, bicycle-following model was established based on the stimulus-response between the front and rear bicycles with the hypothesis that bicycle runs on a virtual lane. In the early study, Hossain M. [1] proposed a mixed traffic network simulation model (MIXNETSIM model). In this model, the bicycle movement was decomposed into two-dimensional coordinates, and then the car-following model was used to analyse the longitudinal following movement of the bicycle. It would provide a theoretical basis for the study on the longitudinal motion of bicycle under the condition of cyclist's overtaking, avoiding, etc. In the later research, Zhao De [2] and Wang Huadong et al. [3] found that the following phenomenon of bicycle was not obvious when road traffic density was low and the following behaviour appeared only under the condition of limited travel space. The cellular automaton model is a widely used physical model in discrete time-space. It can be used to simulate the complex traffic state through simple rules. 
Aiming at the conflict between vehicles and bicycles in a mixed environment, Jia Bin [4] and Zhao Xiaomei [5] respectively established coupling and multi-value cellular automaton model and simulated the situation of mutual interference between vehicles and bicycles. Speed, position, and other characteristics of the bicycle can be expanded to a vector in the simulation model, and it can describe the track of bicycle and its movement characteristics of flexibility, freedom, and mobility. Huang Ling [6], Liang Xiao [7], Chen Da Fei [8] and other scholars used the psychology of "social force" or "psychological force" thought, respectively established the subjective optimization model of NCB (Normative Cyclist Behavioral) theory, micro-perception model. The above studies were the theoretical foundation for the study of bicycle traffic micro-behaviour. In addition, some researchers studied the movement entity in the pedestrian-bicycle mixed traffic environments. To solve the conflict phenomenon on a shared-use sidewalk, Chen Jun, Xie Zhiquan [9] and Deng Jianhua [10] established the shared road traffic conflict model and multi-scale cellular automaton model to describe the motion characteristics of the traffic entity under the condition of sharing traffic. They can help to the design, planning, and management of the pedestrian-bicycle shared road.

Although the car-following model, cellular automata model and simulation model have many advantages in the microcosmic study of bicycle traffic to a certain extent, there are also some problems. The car-following model can only describe the situation where there is no overtaking on a single lane, and the bicycle is regarded as a kind of smart car in the model which is not entirely consistent with the motion characteristics of bicycles. The bicycle lane is artificially divided in the cellular automaton model, and the running space is discretized into grid structure. However, this model lacks the description of individual characteristics such as age, gender, pedal frequency, etc., and the influence of individual characteristics for bicycles cannot be added into the model. The simulation model, such as non-motor vehicle vector field model and the behaviour model of psychological or social field has studied the microscopic behaviour of bicycles from many perspectives. Nevertheless, most of the existing models focus on the interference of static obstacles, and the interaction between dynamic traffic objects and target bicycle in complex traffic environment was not taken into consideration. At the same time, the research on mutual interference of moving objects in mixed traffic environment is relatively rare, and the pre-existing results are relatively rough only to provide some macroscopic descriptions (road capacity, level of service and bicycle traffic flow on vehicle-bicycle or pedestrian-bicycle mixed traffic sections). The internal microscopic mechanism of the interaction between bicycles and pedestrians could not be explained in those pre-existing research results. Consequently, the phasefield coupling theory has been developed in the paper to comprehensively analyse the reduction method of the traffic phase. Feature vectors of different types of cyclists for intention identification are extracted from contracted pedestrian-bicycle traffic phase using the adaptive genetic algorithm based on information entropy. The cyclist's intention identification model has been also established here based on the evidence theory, which is of great significance for studying pedestrian-bicycle interactive security system. The present research takes an important role in promoting the development of green and healthy travelling.

\section{METHODOLOGY}

\subsection{Simulation of pedestrian-bicycle traffic phase}

Phase-field coupling theory is used to simulate the evolution process of phase microstructure [11]. The concept of pedestrian-bicycle traffic phase is proposed for the pedestrian-bicycle mixed traffic system based on Ginzburg-Landau theory of phase transitions and the concept of traffic situation in literature [12].

\section{Definition of pedestrian-bicycle traffic phase}

As shown in Figure 1, pedestrian-bicycle mixed section was divided into several virtual lanes according to the moving direction of the traffic entity. Compared to the pedestrians, bicycles and their cyclists cover much more area. Therefore, according to the maximal density and external contour size of bicycles and its cyclists in reference [13], the area was virtually divided into three different ones including left, middle and right one with the width of 1 metre. Generally, the density of a cyclist is 0.65 per square metre; a single bicycle and its cyclist cover about 1.56 square metres. The maximal length of a bicycle outer contour is about 1.9 metres, while the maximal width is about 0.6 metres. The interest-sensitive area of a cyclist was divided into eight subareas including right front, right rear, next right rear, front, back, left front, left rear, next left rear area, and the location of front axle of target bicycle is used as the initial position. Due to the limitations of the natural senses of cyclists, this present study only defines the interest-sensing areas, which can influence the riding process and can be perceived by the perception system of the cyclist. The remarks of some symbols can be noted in Table 1.

Mathematical expression of pedestrian-bicycle traffic phase

Actually, the cyclist's perception of the interest area is fuzzy and inaccurate. The form of the fuzzy logic method is to carry out approximate reasoning using the linguistic variable. It is appropriate for describing 


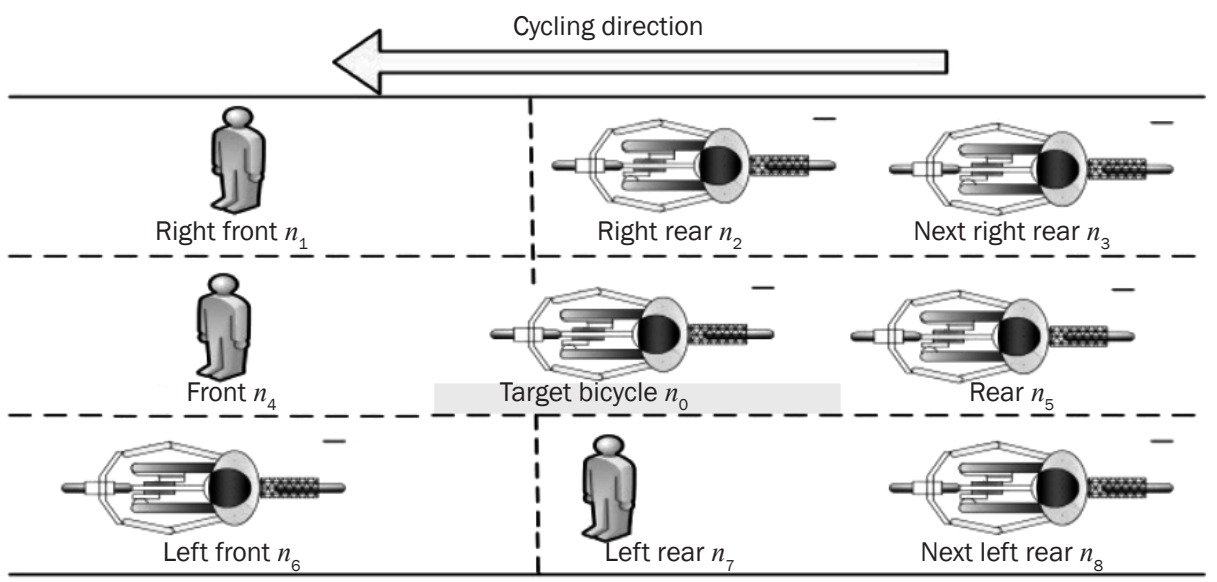

Figure 1 - Diagram of a traffic phase

Table 1 - Symbol description

\begin{tabular}{||l|l||}
\hline \multicolumn{1}{|c|}{ Symbol } & \multicolumn{1}{c||}{ Remarks } \\
\hline \hline$n_{0}$ & Target bicycle and its cyclist. \\
\hline$n_{i}(i=1,2,3,4,5,6,7,8)$ & Traffic entity in each orientation. \\
\hline$v_{n_{i}}(i=1,2,3,4,5,6,7,8)$ & The speed of traffic entity and the target bicycle. \\
$\Delta d_{i}(i=1,2,3,4,5,6,7,8)$ & $\begin{array}{l}\text { Relative distance between the traffic entity in each orientation and the } \\
\text { target bicycle. }\end{array}$ \\
\hline$\Delta v_{i}=v_{n_{i}}-v_{n 0}(i=1,2,3,4,5,6,7,8)$ & $\begin{array}{l}\text { Relative speed between the traffic entity in each orientation and the target } \\
\text { bicycle. }\end{array}$ \\
\hline$F_{i} \in\{$ pedestrian, bicycle $(i=1,2,3,4,5,6,7,8)$ & Types of traffic entities. \\
\hline
\end{tabular}

the subjective judgment process of the cyclist movement [14-16]. The field intensity was calculated based on the fuzzy logic method, and it described the attraction or repulsion interaction between the target bicycle and each traffic entity in the target cyclist's interest area. The "force" was used to describe field intensity, and the greatest repulsion field intensity $\left(P E_{i}, i=1,2,3\right)$ and the greatest attraction field intensity $\left(N E_{i}, i=1,2,3\right)$ are represented by -1 and 1 , respectively. The strong and weak force of different field intensity is represented by a real number of intervals (Table 2 ).

Three factors including traffic entity type, relative speed between every traffic entity and target bicycle and relative distance were comprehensively considered in each interest subarea of the target cyclist, and the fuzzy logic method was used to reasonably sieve the force.
As described above, traffic entities type, relative speed, and relative distance represent three different input variables, and the force of field intensity represents the output variable. The fuzzy sets and the membership grade of the relative speed and distance were computed using the fuzzy logic method in the reference [16]. Due to space limitation, the effect field of the interest-sensitive area will be abbreviated in the following paper, as shown in Table 3.

When the target bicycle is located in the middle area, in Figure 1, the pedestrian-bicycle traffic phase of the target bicycle and its cyclist $n_{0}$ can be expressed by: $E_{n_{0}}^{0}=[\mathrm{LFEF}, \mathrm{LREF}, \mathrm{NLREF}, \mathrm{FEF}, \mathrm{REF}, \mathrm{RFEF}, \mathrm{RREF}$, $\mathrm{NRREF}]=\left[E_{n 0}^{z q}, E_{n 0}^{z h}, E_{n 0}^{z h}, E_{n 0}^{q}, E_{n 0}^{h}, E_{n 0}^{y q}, E_{n 0}^{y h}, E_{n 0}^{y c h}\right]$.

When the target bicycle was located in the left or right area of the pedestrian-bicycle mixed traffic sections, the derivation process of the pedestrian-bicycle traffic phase was similar as the middle one.

Table 2 - Force corresponding to different field intensity

\begin{tabular}{|c|c|c|c|c|c|c|c|}
\hline $\begin{array}{c}\text { Field } \\
\text { intensity }\end{array}$ & $\begin{array}{c}\text { Strong } \\
\text { repulsion } \\
\text { field intensity } \\
P E_{1}\end{array}$ & $\begin{array}{c}\text { Medium } \\
\text { repulsion } \\
\text { field intensity } \\
P E_{2}\end{array}$ & $\begin{array}{c}\text { Weak } \\
\text { repulsion } \\
\text { field intensity } \\
P E_{3}\end{array}$ & $\begin{array}{c}\text { Zero } \\
\text { field } \\
\text { intensity } \\
Z \\
\end{array}$ & $\begin{array}{c}\text { Weak } \\
\text { attraction } \\
\text { field intensity } \\
N E_{1}\end{array}$ & $\begin{array}{c}\text { Medium } \\
\text { attraction } \\
\text { field intensity } \\
N E_{2}\end{array}$ & $\begin{array}{c}\text { Strong } \\
\text { attraction } \\
\text { field intensity } \\
N E_{3}\end{array}$ \\
\hline Force & {$[-1,-0.8)$} & {$[-0.8,-0.4)$} & {$[-0.4,0)$} & 0 & $(0,0.3]$ & $(0.3,0.7]$ & $(0.7,1]$ \\
\hline
\end{tabular}


Table 3 - Abbreviation for effect field of the interest-sensitive area

\begin{tabular}{||l|c||}
\hline \multicolumn{1}{|c|}{$\begin{array}{c}\text { The effect field of the } \\
\text { interest-sensitive area }\end{array}$} & Abbreviation \\
\hline \hline Left front effect field & LFEF \\
\hline Left rear effect field & LREF \\
\hline Next left rear effect field & NLREF \\
\hline Front effect field & FEF \\
\hline Rear effect field & REF \\
\hline Right front effect field & RFEF \\
\hline Right rear effect field & RREF \\
\hline Next right rear effect field & NRREF \\
\hline
\end{tabular}

Simplification of complex pedestrian-bicycle traffic phase

The field intensity of traffic entity in each subarea for the target cyclist can be judged by the force. The intensity of repulsion and attraction field using the fuzzy logic were denoted as "-" and “+”, respectively. According to the field intensity of the front-side traffic entity and the rear-side traffic entity interacting with the target bicycle, the interaction field intensity between the target bicycle and the area of the target bicycle can be obtained. The fuzzy inference rule is shown in Table 4. The interaction field intensity between the target bicycle and other areas of the non-target bicycle can be obtained with the similar derivation process.

When the target bicycle is located in the left or right area of the pedestrian-bicycle mixed traffic sections, the target bicycle and its cyclist are influenced by the adjacent and separated area. When the traffic entity changes the trajectory in the separated area, the traffic entity of the adjacent area will be affected and the target cyclist's intention is disturbed at the same time.

In summary, the complex pedestrian-bicycle traffic phase can be reduced to 16 types, as shown in Figure 2.

\subsection{Cyclist's intention identification in pedestrian-bicycle mixed environment}

The cyclist's intention is to play an important role in the change of pedestrian-bicycle traffic phase. It is important for the study of the pedestrian-bicycle interactive security system to accurately identify the cyclist's

Table 4 - Fuzzy inference rules of regional action field intensity

\begin{tabular}{||c|l|l|c||}
\hline $\begin{array}{c}\text { Rule } \\
\text { numbers }\end{array}$ & The field intensity of front area traffic entity & The field intensity of rear area traffic entity & $\begin{array}{c}\text { The field intensity } \\
\text { of the area }\end{array}$ \\
\hline \hline $1-4$ & Strong repulsion or Medium repulsion & Strong repulsion or Medium repulsion & Repulsion \\
\hline $5-10$ & Strong repulsion or Medium repulsion & Weak repulsion or Zero or Weak attraction & Repulsion \\
\hline $11-14$ & Strong repulsion or Medium repulsion & Strong attraction or Medium attraction & Repulsion \\
\hline $15-20$ & Weak repulsion or Zero or Weak attraction & Strong repulsion or Medium repulsion & Zero \\
\hline $21-29$ & Weak repulsion or Zero or Weak attraction & Weak repulsion or Zero or weak Attraction & Attraction \\
\hline $30-35$ & Weak repulsion or Zero or Weak attraction & Strong attraction or Medium attraction & Atrong repulsion or Medium repulsion \\
\hline $36-39$ & Medium attraction or Strong attraction & Straction & Attraction \\
\hline $40-45$ & Medium attraction or Strong attraction & Weak repulsion or Zero or Weak attraction & Medium attraction or Strong attraction \\
\hline $46-49$ & Medium attraction or Strong attraction &
\end{tabular}

Cycling direction

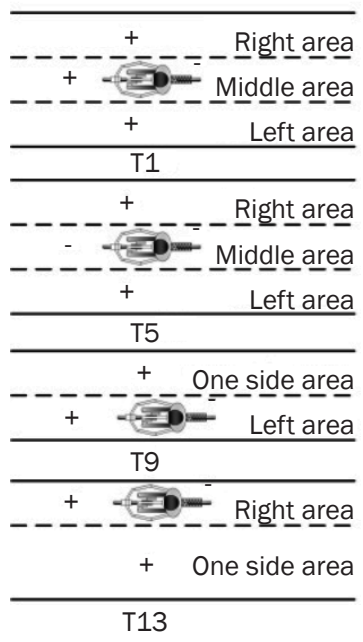

$\mathrm{T} 13$

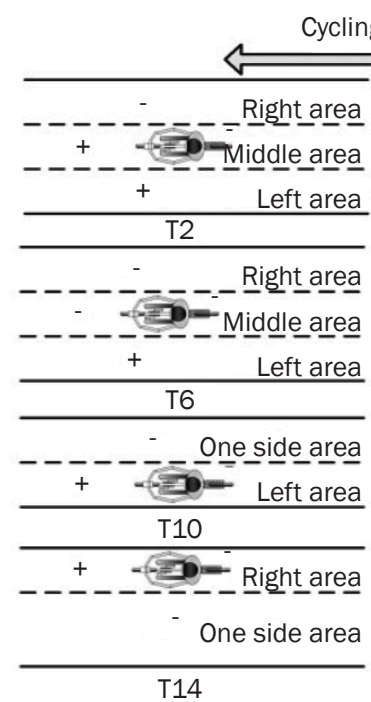

T14

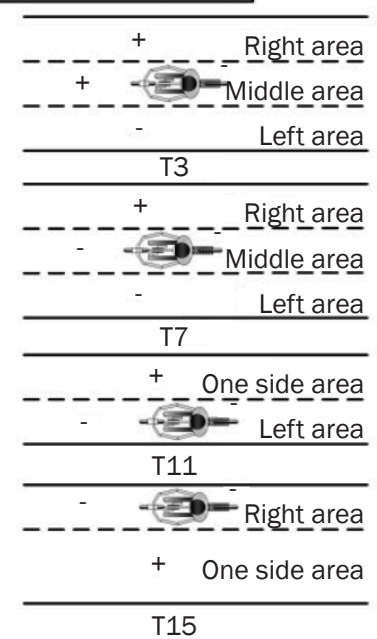

T15

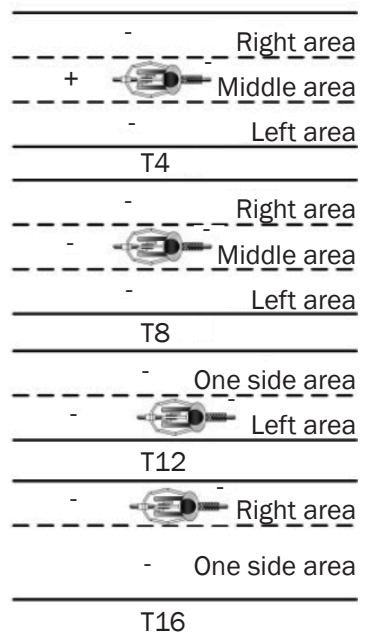

T16

Figure 2 - Simple pedestrian-bicycle traffic phase 
intention. Here, Dempster-Shafer evidence theory was applied into establishing the cyclist's intention identification model in a pedestrian-bicycle mixed environment.

\subsubsection{Dempster-Shafer evidence theory}

The basis of evidence theory combines the evidence with the update of belief function; the uncertainty information was described with the concept of identification framework, probability assignment function, belief function, etc. [17].

Firstly, evidence identification framework and basic belief assignment function should be determined. The evidence identification framework $D$ is a complete set of all possible answers related to an uncertainty problem, and then any proposition corresponds to a subset of $D$.

The basic belief assignment function $M$ is a mapping from set $2^{D}$ to $[0,1]$. If any proposition $A$ belongs to $D$, which is expressed as formula " $A \subseteq D$ ", and satisfies the condition $M(\Phi)=0$ and $\sum_{B \subseteq A} M(A)=1$, then $M(A)$ is the basic belief assignment function for proposition $A$ on set $2^{D}$. The degree of belief $\operatorname{Bel}(A)=\sum_{B \subseteq A} A(B), \forall A \subseteq D$ (the sum of basic probability assignment in all subsets $B$ of proposition $A$ ). $M(\Phi)$ shows that no proposition has been assigned, and it is only used to represent the position information.

Assuming that the belief assignment functions of the propositions $A$ and $B$ are $M(A)$ and $M(B)$, respectively, then the synthesized basic belief assignment function is

$$
\begin{aligned}
& M(C)=\left\{\begin{array}{l}
\frac{\sum_{A \cap B=C} M(A) M(B)}{1-K} \\
0 \\
\forall C \subset U, C \neq \Phi, \quad K=\sum_{A \cap B=\Phi} M(A) M(B), C=\Phi
\end{array}\right.
\end{aligned}
$$

If $K=1$, propositions $A$ and $B$ cannot be synthesized; otherwise, they can be realized.

Multiple evidence combination rules are introduced next. Let $M_{1}, M_{2}, \ldots, M_{n}$ be the basic belief assignment in the same identification framework, and the corresponding focal elements are $A_{1}, A_{2}, \ldots, A_{n}$, respectively, then the combination formula of multiple pieces of evidence can be obtained as follows:

$$
\begin{aligned}
& M(A)=(1-K)^{-1} \sum_{A_{1} \cap A_{2} \cap \ldots \cap A_{n}} M_{1}\left(A_{1}\right) M_{2}\left(A_{2}\right) \ldots M_{n}\left(A_{n}\right) \\
& K=\sum_{A 1 \cap \ldots \cap A 2=0} M_{1}\left(A_{1}\right) M_{2}\left(A_{2}\right) \ldots M_{n}\left(A_{n}\right)
\end{aligned}
$$

Finally, the decision rules can be found according to the following assumption.

Assuming that $\exists A_{1}, A_{2} \subset D$, if the condition

$$
\left\{\begin{array}{l}
M\left(A_{1}\right)-M\left(A_{2}\right)>\varepsilon_{1} \\
M(D)<\varepsilon_{2} \\
M\left(A_{1}\right)>M(D)
\end{array}\right.
$$

is satisfied, then $A_{1}$ is the judgement result. Here, $\varepsilon_{1}$ and $\varepsilon_{2}$ are pre-set thresholds in the condition, respectively.

\subsubsection{Model establishment}

This paper is to identify the cyclist's intentions in a pedestrian-bicycle mixed environment. It is assumed that there are $n$ kinds of cyclist's intentions within the identification framework. In Figure 3, the $k$ characteristic parameters extracted by the adaptive genetic algorithm based on the information entropy are expressed as $E_{1}, E_{2}, \ldots, E_{k^{*}} M_{1}\left(I_{i}\right), M_{2}\left(I_{i}\right), \ldots$, respectively. $M_{k}\left(I_{i}\right)$ $(i=1,2, \ldots, n)$ are the basic belief assignment functions corresponding to $k$ characteristic parameters for the intention $I_{i}$.

Since the combination of multiple pieces of evidence was independent of order, the results from the evidence synthesis shown in Figure 4 are recursively calculated by random synthesis of multiple evidence elements. Firstly, the probability assignment of the evidence element was initialized. Secondly, the basic belief assignment function of the evidence element was calculated. After that a new evidence set was obtained using the evidence combination rules. Finally, the result of the combination is judged by the degree of belief, and the cyclist's intention with the maximal degree of belief was chosen as an alternative intention. It can be seen from the above, when the judgment decision

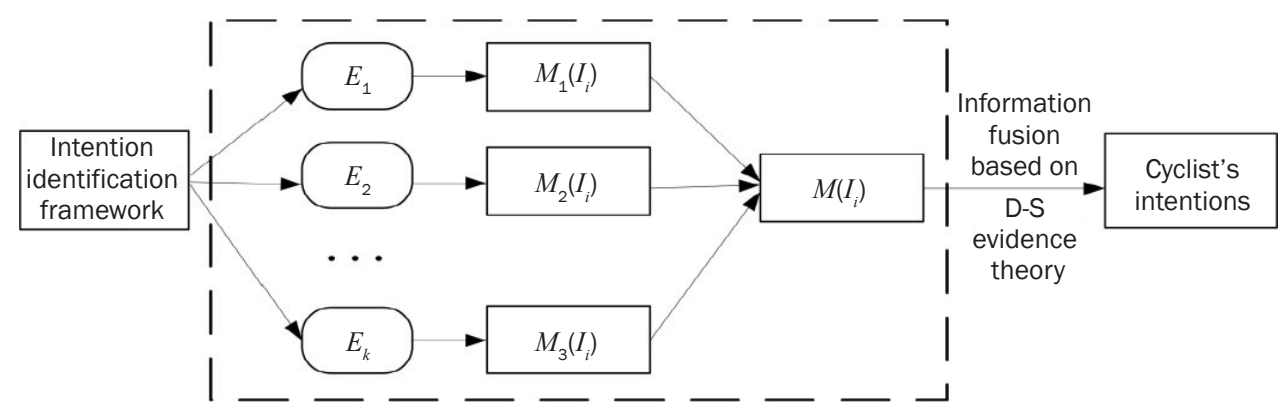

Figure 3 - Cyclist's intention identification model based on D-S evidence theory 

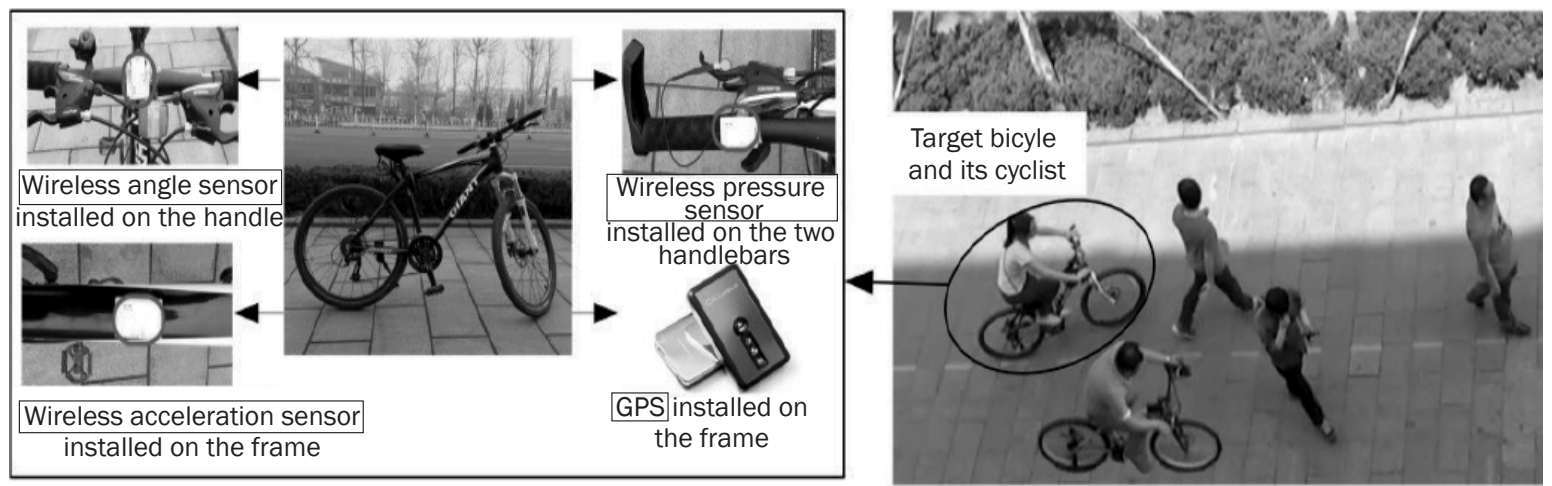

Figure 4 - Dynamic acquisition system for complex information of bicycle and its cyclist

value of the cyclist's intention exceeds $\varepsilon$, the intention is judged to be an intention of the target cyclist in the pedestrian-bicycle mixed environment.

\subsubsection{Data acquisition and processing}

\section{Experimental installation}

On the urban pedestrian-bicycle mixed sections, a dynamic acquisition system for complex information of bicycle and its cyclist (as shown in Figure 4, including Psylab Human Factors engineering experiment wireless sensors, GPS, Laptop) and video collection system (HD camera and tripod) were used to collect and process the experimental data. In addition, the software SPSS21.0, PsyLAB_Installer, VideoStudio 10.0. were applied in the experiment.

\section{Experimental location and road conditions}

The experiment of data collection was carried out in good weather. The mixed pedestrian-bicycle section in Huaguang Road (between north Xiwu Road and Liuquan Road in Zhangdian District of Zibo city) has been selected as the experimental section. These sections serve the pedestrians and bicycles. There is no interference vehicle on this section during the experiment process for getting more accurate data. The length of the test section is $3,450 \mathrm{~m}$. Sites $100 \mathrm{~m}$ away from the intersections were selected as start and end points of the experimental section. In Figure 5, the flagpole is

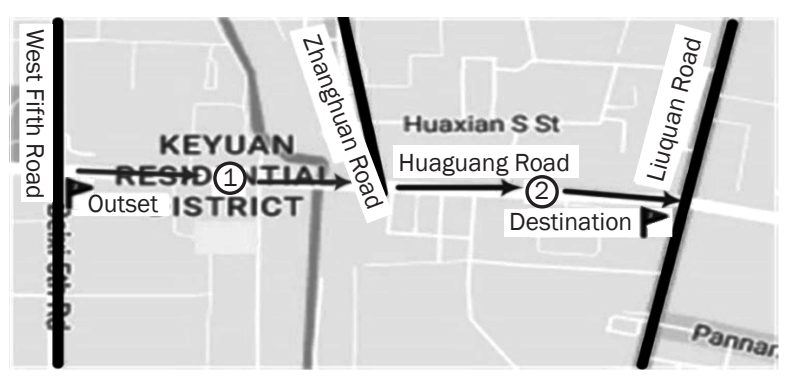

Figure 5 - Experimental route used for marking. Section 1 has been set between the sections of West Fifth Road and Zhanghuan Road, and Section 2 has been set between the sections of Zhanghuan Road and Liuquan Road.

\section{Experimental contents}

First, the cyclist was recruited at the starting point of a specified experimental route. The type of cyclist (conservative, steady, radical) was determined by the questionnaire. Secondly, GPS has been calibrated on the bicycle, video acquisition system and dynamic comprehensive information collection system were used in experiments and the instruments were conducted normally during the process of experiment. Finally, cyclists ride naturally on the experimental section; the relevant experimental data were recorded by a video collection system and a dynamic information collection system in real time, and data were exported and saved in the terminal of an experimental route.

\section{Subjects of experiment}

A hundred subjects (including 52 male and 48 female cyclists, at the age from 10 to 65 ) were randomly selected according to the above experimental contents, as depicted in Table 5. The results of the questionnaire test (cyclists' psychological questionnaire is shown in Table 6) on 100 cyclists' psychological surveys are shown in Table 5. This study was approved by the local institutional review board and written informed consent was obtained from all the participants.

Table 5 - Test results of cyclist's psychological questionnaire

\begin{tabular}{|l||c|c|c||}
\hline Cyclist type & Conservative & Steady & Radical \\
\hline Real scene test results & 24 & 46 & 30 \\
\hline
\end{tabular}

Note: The homogeneity, reliability and validity of all the questionnaires were evaluated by SPSS21.0 software, and the internal consistency Cronbach's Alpha coefficient was $0.893>0.8$ Approximately $94 \%$ of the problem scores in the questionnaire were significantly correlated with the total score at the significance level of 0.05 and 0.01 . This shows that the questionnaire has a higher homogeneity and better content validity. 
Table 6 - Cyclists' psychological questionnaire

\begin{tabular}{||l|}
\hline 1. Age: \\
\hline 2. Job: \\
\hline 3. Gender: (1) Male (2) Female \\
\hline 4. Education background: (1) Junior high school, (2) Senior middle school, (3) University. \\
\hline $\begin{array}{l}\text { 5. When you ride, are you going to be in a hurry? } \\
\text { (1) Never, (2) Rarely, (3) Sometimes, (4) Often. }\end{array}$ \\
\hline
\end{tabular}

6. Will you accelerate through the intersection during the signal transition?

(1) Never, (2) Rarely, (3) Sometimes, (4) Often.

7. When you ride, are you riding fast?

(1) Never, (2) Rarely, (3) Sometimes, (4) Often.

8. Will you pay attention to the surrounding bicycles or pedestrians during your riding?

(1) Absolutely, (2) Sometimes, (3) Rarely, (4) Never

9. Are you upset when the current rider is slow?

(1) Never, (2) Rarely, (3) Sometimes, (4) Often.

10. When you are riding, do you always want to bypass pedestrians?

(1) Never, (2) Rarely, (3) Sometimes, (4) Often.

11. There are vehicles occupying the road in the slow area.

(1) Parking, (2) Deceleration, (3) Accelerated detour, (4) Straight riding

12. Do you always follow the traffic entity in the process of riding?

(1) Never, (2) Rarely, (3) Sometimes, (4) Often.

13. When you ride on a familiar road, do you relax your alert?

(1) Never, (2) Rarely, (3) Sometimes, (4) Often.

14. Will you check the condition of the bicycle such as brakes and the tire pressure before riding?

(1) Absolutely, (2) Sometimes, (3) Rarely, (4) Never

15. Do you brake frequently or accelerate when you are travelling?

(1) Absolutely, (2) Sometimes, (3) Rarely, (4) Never

16. When you are riding a bicycle, do you make calls or listen to music, etc.

(1) Never, (2) Rarely, (3)Sometimes, (4) Often.

17. Will you carry people while riding?

(1) Never, (2) Rarely, (3) Sometimes, (4) Often.

18. Will you look around while you are riding?

(1) Absolutely, (2) Sometimes, (3) Rarely, (4) Never

19. Will you leave the handlebar with one hand or both hands while you are riding?

(1) Never, (2) Rarely, (3) Sometimes, (4) Often.

20. Will you increase your speed without realizing while you are riding?

(1) Never, (2) Rarely, (3) Sometimes, (4) Often.

21. Do you feel anxious when the current pedestrians and bicycles are more likely to cause traffic congestion?

(1) Never, (2) Rarely, (3) Sometimes, (4) Often.

22. Will you change to a motorway or speed up when you can ensure safe riding?

(1) Never, (2) Rarely, (3) Sometimes, (4) Often.

23. Will you intersperse with the gap when the riding space allows?

(1) Never, (2) Rarely, (3) Sometimes, (4) Often.

24. Are you in a hurry when you are waiting for the traffic signal?

(1) Never, (2) Rarely, (3) Sometimes, (4) Often.

25. Will you overtake during the ride, when the current bicycle is slow?

(1) Never, (2) Rarely, (3) Sometimes, (4) Often.

26. Will you change the riding track arbitrarily when you have enough riding space?

(1) Never, (2) Rarely, (3) Sometimes, (4) Often.

27. Do you feel angry when there are other bicycles that bypass you?

(1) Never, (2) Rarely, (3) Sometimes, (4) Often.

28. Avoidance of obstacles

(1) Avoid in advance, (2) Avoid normally, (3) Not sure, (4) Very urgent avoidance

Note:

1) The number in parentheses is the score of the corresponding option.

2) Scores from questions 5 to 28 are introduced next. Conservative cyclists scored 28 to 46 , steady cyclists scored 47 to 68 , and radical cyclists scored 69 to 88 . 
Table 7 - Partial movement characteristics of bicycle and its cyclist

\begin{tabular}{|c|c|c|c|c|c|c|c|c|}
\hline \multicolumn{2}{|c|}{ Data } & \multirow{2}{*}{$\frac{\text { Symbol }}{\mathrm{A}_{11}}$} & \multicolumn{2}{|r|}{ Data } & \multirow{2}{*}{$\begin{array}{c}\text { Symbol } \\
A_{31}\end{array}$} & \multicolumn{2}{|c|}{ Data } & Symbol \\
\hline \multirow{8}{*}{ 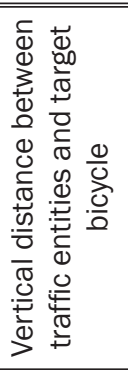 } & Right front & & \multirow{8}{*}{ 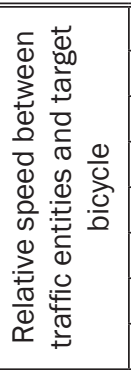 } & Right front & & \multirow{8}{*}{ 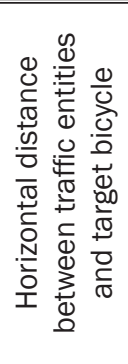 } & Right front & $A_{21}$ \\
\hline & Right rear & $A_{12}$ & & Right rear & $\mathrm{A}_{32}$ & & Right rear & $A_{22}$ \\
\hline & Next right rear & $A_{13}$ & & Next right rear & $A_{33}$ & & Next right rear & $A_{23}$ \\
\hline & Front & $\mathrm{A}_{14}$ & & Front & $A_{34}$ & & Front & $\mathrm{A}_{24}$ \\
\hline & Rear & $A_{15}$ & & Rear & $A_{35}$ & & Rear & $A_{25}$ \\
\hline & Left front & $A_{16}$ & & Left front & $A_{36}$ & & Left front & $A_{26}$ \\
\hline & Left rear & $\mathrm{A}_{17}$ & & Left rear & $A_{37}$ & & Left rear & $\mathrm{A}_{27}$ \\
\hline & Next left rear & $\mathrm{A}_{18}$ & & Left rear side & $A_{38}$ & & Next left rear & $A_{28}$ \\
\hline \multicolumn{2}{|c|}{$\begin{array}{l}\text { Target bicycle } \\
\text { displacement }\end{array}$} & $\mathrm{A}_{4}$ & \multicolumn{2}{|c|}{ Target bicycle speed } & $A_{5}$ & \multicolumn{2}{|c|}{$\begin{array}{l}\text { Target bicycle accelera- } \\
\text { tion }\end{array}$} & $A_{6}$ \\
\hline \multicolumn{2}{|c|}{$\begin{array}{l}\text { Target bicycle front wheel } \\
\text { steering angle }\end{array}$} & $\mathrm{B}_{1}$ & \multicolumn{2}{|c|}{ Tic frequency } & $\mathrm{B}_{2}$ & \multicolumn{2}{|c|}{ Brake intensity } & $\mathrm{B}_{3}$ \\
\hline
\end{tabular}

Data processing and feature extraction

The shooting results from the video capture system and the output results from the comprehensive information dynamic collection system were analysed. Based on the above results, the high-reliability characteristics data of bicycle and its cyclist can be obtained. Section 1 was the running adaptation section for the bicycle that was equipped, the data collected from Section 2 were the required study sample ones. The sample data were organized according to one bicycle per second. Some motion characteristics can be found in Table 7.

According to the above data, take the pedestrian-bicycle traffic phase $T_{1}$ as an example. The adaptive genetic algorithm based on the information entropy [18] is used to extract the characteristic $A_{3 i}(i=1,2,3,4,5,6,7,8,9)$ and $B_{j}(i=1,2,3)$ in the pedestrian-bicycle traffic phase $T_{1}$.

The feature data of cyclists' intentions in several other pedestrian-bicycle traffic phases can be extracted using the same method, and the results are omitted due to the limitation of the space.

\section{RESULTS}

\subsection{Model calibration}

The feature parameter of target cyclist's intention is extracted in "Section 2.2.3". In this paper, because of the limited space, the identification of steady cyclist's intention was taken as an example to elaborate on the pedestrian-bicycle traffic phase. Under the condition that the target bicycle is driving stably in the simple pedestrian-bicycle traffic phase $T_{1}$, the characteristic parameter of the cyclist's intention was described in the above "Section 2.2.3". RS denotes the relative speed between traffic entities and target bicycle. Target bicycle front wheel steering angle, tic frequency and brake intensity were represented by $B F A, T P F, P B D$, respectively, in order to facilitate the establishment and analysis of the model.
The intention of different types of cyclists in different traffic phases were divided into eight kinds, as depicted in Table 8.

Table 8 - Cyclist's intention

\begin{tabular}{||l|c||}
\hline \multicolumn{1}{|c|}{ Intention } & Symbol \\
\hline \hline Bypass from the left at an increased speed & $I_{11}$ \\
\hline Bypass from the right at an increased speed & $I_{12}$ \\
\hline Bypass from the left at the same speed & $I_{21}$ \\
\hline Bypass from the right at the same speed & $I_{22}$ \\
\hline Bypass from the left at a decreased speed & $I_{31}$ \\
\hline Bypass from the right at a decreased speed & $I_{32}$ \\
\hline Follow at a decreased speed & $I_{4}$ \\
\hline Stop and wait & $I_{5}$ \\
\hline
\end{tabular}

The eight kinds of intention were deduced from analysing and processing the characteristic parameters. Then, the intention identification framework $D=\left(I_{11}, I_{12}, I_{21}, I_{22}, I_{31}, I_{32}, I_{4}, I_{5}\right)$ and the evidence set $E=(R S, B F A, T P F, P B D)$ can be obtained according to the classification of the cyclist's intention.

In this paper, the data of 60 samples were extracted as the basic data of intention identification model from the experiment in "Section 2.2.3". The basic probability assignment of each element in the evidence set was determined for the identification framework and evidence set, as shown in Table 9, where $O$ denotes the uncertain probability assignment.

\subsection{Model solution}

According to the D-S evidence theory combination rule, this paper identifies the intention of a steady cyclist in a simple pedestrian-bicycle traffic phase based on characterizing the cyclist's intentional feature parameters. At a certain time, corresponding to different sections of each element in the evidence set $E=(R S ; B F A, T P F, P B D)$ in Table 9, the elements from 1 to 12 are divided into four groups of $\left(R S_{1}, R S_{2}\right.$, $\left.R S_{3}\right),\left(B F A_{4}, B F A_{5}, B F A_{6}\right),\left(T P F_{7}, T P F_{8}, T P F_{9}\right)$ and 
Table 9 - Basic probability assignment of each element in the evidence set

\begin{tabular}{|c|c|c|c|c|c|c|c|c|c|c|c|}
\hline \multicolumn{3}{|c|}{ Evidence set } & $I_{11}$ & $I_{12}$ & $I_{21}$ & $I_{22}$ & $I_{31}$ & $I_{32}$ & $I_{4}$ & $I_{5}$ & $O$ \\
\hline \multirow{3}{*}{$\begin{array}{c}R S \\
{[\mathrm{~km} / \mathrm{h}]}\end{array}$} & 1 & {$[10.26,13.17]$} & 0.28 & 0.35 & 0.41 & 0.42 & 0.29 & 0.28 & 0.19 & 0.32 & 0.19 \\
\hline & 2 & {$[13.17,14.41]$} & 0.25 & 0.26 & 0.26 & 0.25 & 0.34 & 0.32 & 0.29 & 0.28 & 0.21 \\
\hline & 3 & {$[14.41,15.60]$} & 0.39 & 0.33 & 0.21 & 0.22 & 0.62 & 0.59 & 0.50 & 0.31 & 0.09 \\
\hline \multirow{3}{*}{$\begin{array}{c}B F A \\
\text { [Newton] }\end{array}$} & 4 & {$[14.95,23.65]$} & 0.49 & 0.48 & 0.32 & 0.33 & 0.25 & 0.24 & 0.23 & 0 & 0.19 \\
\hline & 5 & {$[23.65,26.17]$} & 0.32 & 0.34 & 0.30 & 0.28 & 0.20 & 0.22 & 0.18 & 0 & 0.38 \\
\hline & 6 & {$[26.17,37.91]$} & 0.18 & 0.21 & 0.41 & 0.40 & 0.43 & 0.41 & 0.35 & 0.09 & 0.32 \\
\hline \multirow{3}{*}{$\begin{array}{c}T P F \\
{[\mathrm{circle} / \mathrm{min}]}\end{array}$} & 7 & {$[20,25]$} & 0.09 & 0.09 & 0.58 & 0.56 & 0.15 & 0.16 & 0.46 & 0 & 0.33 \\
\hline & 8 & {$[25,30]$} & 0.35 & 0.35 & 0.48 & 0.46 & 0.28 & 0.27 & 0.13 & 0 & 0.12 \\
\hline & 9 & {$[30,35]$} & 0.72 & 0.62 & 0.21 & 0.20 & 0.23 & 0.21 & 0.28 & 0 & 0.07 \\
\hline \multirow{3}{*}{$\begin{array}{c}P B D \\
{[\mathrm{~m}]}\end{array}$} & 10 & {$[0.93,1.46]$} & 0.56 & 0.13 & 0.33 & 0.35 & 0.23 & 0.20 & 0.15 & 0 & 0.11 \\
\hline & 11 & {$[1.46,2.23]$} & 0.43 & 0.51 & 0.38 & 0.37 & 0.16 & 0.17 & 0.12 & 0 & 0.19 \\
\hline & 12 & {$[2.23,3.00]$} & 0 & 0.36 & 0.45 & 0.46 & 0.32 & 0.29 & 0.45 & 0.49 & 0.06 \\
\hline
\end{tabular}

$\left(P B D_{10}, P B D_{11}, P B D_{12}\right)$. These elements of four different sets are combined; especially, the elements in the same set cannot be realized. According to the calculation of Formulas 2 and 3 , the inconsistency factor between $P B D_{1}$ and $B F A_{4}$ is $K_{1,4}=0.3409$. The basic probability assignment function values of the fusion of the evidences $R S_{1}$ and $B F A_{4}$ are $M_{1,4}\left(I_{11}\right)=0.4163$, $M_{1,4}\left(I_{12}\right)=0.4562, \quad M_{1,4}\left(I_{21}\right)=0.1602, \quad M_{1,4}^{1,4}\left(I_{22}\right)=0.1826$, $M_{1,4}\left(I_{31}\right)=0.2103, M_{1,4}\left(I_{32}\right)=0.1903, M_{1,4}\left(I_{4}\right)=0.1096$ and $M_{1,4}\left(I_{5}\right)=0 . T P F_{7}$ is fused after the fusion of $R S_{1}$ and $B F A_{4}$, and the inconsistency factor of $R S_{1}, B F A_{4}$ and $\mathrm{TPF}_{7}$ is $K_{1,4,7}=0.368$. The basic probability assignment function values of the fusion of the evidences $R S_{1}, B F A_{4}$ and $\mathrm{TPF}_{7}$ are $M_{1,4,7}\left(I_{11}\right)=0.05269, M_{1,4,7}\left(I_{12}\right)=0.04869$, $M_{1,4,7}\left(I_{21}\right)=0.10394, \quad M_{1,4,7}\left(I_{22}\right)=0.14273, \quad M_{1,4,7}\left(I_{31}\right)=$ $0.04862, \quad M_{1,4,7}\left(I_{32}\right)=0.03632, \quad M_{1,4,7}\left(I_{4}\right)=0.03698$ and $M_{1,4,7}\left(I_{5}\right)=0$. The evidence elements $P B D_{10}, R S_{1}$, $B F A_{4}$ and $T P F_{7}$ are fused in different groups. The inconsistency factor of $R S_{1}, B F A_{4}, T P F_{7}$ and $P B D_{10}$ is $K_{1,4,7,10}=0.03992$. The basic probability assignment function values of the fusion of the evidences $R S_{1}, B F A_{4}, T P F_{7}$ and $P B D_{10}$ are $M_{1,4,7,10}\left(I_{11}\right)=0.00382$, $M_{1,4,7,10}\left(I_{12}\right)=0.00494, M_{1,4,7,10}\left(I_{21}\right)=0.06279, M_{1,4,7,10}\left(I_{22}\right)=$ $0.06426, \quad M_{1,4,7,10}\left(I_{31}\right)=0.00249, \quad M_{1,4,7,10}\left(I_{32}\right)=0.00352$, $M_{1,4,7,10}\left(I_{4}\right)=0.00196$ and $M_{1,4,7,10}\left(I_{5}\right)=0$. The fusion between the other evidence elements in the four groups of numbers is the same as the one among the evidence elements $R S_{1}, B F A_{4}, T P F_{7}$ and $P B D_{10}$.

According to the fusion decision rules, the threshold was set as $\varepsilon_{1}=0.5$ and $\varepsilon_{2}=1$. With the fusion of multiple pieces of evidence, the certainty of identification was improved gradually. After combining several evidences with each other, the degree of the basic belief for $I_{11}, I_{12}, I_{21}, I_{22}, I_{31}, I_{32}, I_{4}$, and $I_{5}$ are $\operatorname{Bel}\left(I_{11}\right)=1.74896, \quad \operatorname{Bel}\left(I_{12}\right)=1.75236, \quad \operatorname{Bel}\left(I_{21}\right)=2.74605$, $\operatorname{Bel}\left(I_{22}\right)=1.92014, \quad \operatorname{Bel}\left(I_{31}\right)=1.56726, \quad \operatorname{Bel}\left(I_{32}\right)=1.60382$, $\operatorname{Bel}\left(I_{4}\right)=1.46579$, and $\operatorname{Bel}\left(I_{5}\right)=1.36951$, respectively. The intention of a steady cyclist is $I_{21}$ in the pedestrian-bicycle traffic phase $T_{1}$.
D-S evidence theory was also used to identify the intention of the target cyclist from several other pedestrian-bicycle traffic phases. Finally, the target cyclist's intention could be obtained from 16 kinds of pedestrian-bicycle traffic phases, as shown in Table 10.

\section{DISCUSSION}

Field experiments verification

The remaining 40 sample data were used here to experimentally validate the cyclists' intention identification model. On the basis of the data captured from the video, the validity and reliability of the identification model can be verified through identifying the target cyclists' intention in real-time and contrasting with the target cyclist' behaviour in the video. According to the comparison, the model parameters were revised and the cyclists' intention identification model was built in the pedestrian-bicycle mixed traffic based on the phase field coupling theory.

As shown in Figure 6, the fitness of the identification result with the observation result is high, and the accuracy of identification is about $90 \%$.

\section{Simulation verification}

According to the experiment of pedestrian-bicycle mixed sections, the cyclists' intention identification model has been built respectively based on the phase field coupling theory and D-S evidence theory. This simulation program is used to simulate the macroscopic rule (such as flow, density, and speed) and the microscopic rule (such as velocity, acceleration, displacement) and to verify the results. The effect of the cyclist's intention identification is validated by comparing the validation results with the experimental situation on pedestrian-bicycle mixed sections.

The validation is shown in the following figures. The distribution of the bicycle traffic flow, density and mean velocity in a pedestrian-bicycle mixed 
Table 10 - Different cyclist's intentions identification in pedestrian-bicycle traffic phase

\begin{tabular}{|c|c|c|c|c|c|}
\hline Traffic phase & Cyclist types & Riding intention & Traffic phase & Cyclist types & "Riding intention \\
\hline \multirow{3}{*}{$T_{1}$} & Conservative & $I_{32}$ & \multirow{3}{*}{$T_{9}$} & Conservative & $I_{31}$ \\
\hline & Steady & $I_{21}$ & & Steady & $I_{22}$ \\
\hline & Radical & $I_{12}$ & & Radical & $I_{12}$ \\
\hline \multirow{3}{*}{$T_{2}$} & Conservative & $I_{4}$ & \multirow{3}{*}{$T_{10}$} & Conservative & $I_{5}$ \\
\hline & Steady & $I_{21}$ & & Steady & $I_{4}$ \\
\hline & Radical & $I_{12}$ & & Radical & $I_{12}$ \\
\hline \multirow{3}{*}{$T_{3}$} & Conservative & $I_{32}$ & \multirow{3}{*}{$T_{11}$} & Conservative & $I_{5}$ \\
\hline & Steady & $I_{22}$ & & Steady & $I_{4}$ \\
\hline & Radical & $I_{11}$ & & Radical & $I_{12}$ \\
\hline \multirow{3}{*}{$T_{4}$} & Conservative & $I_{5}$ & \multirow{3}{*}{$T_{12}$} & Conservative & $I_{5}$ \\
\hline & Steady & $I_{4}$ & & Steady & $I_{4}$ \\
\hline & Radical & $I_{21}$ & & Radical & $I_{21}$ \\
\hline \multirow{3}{*}{$T_{5}$} & Conservative & $I_{31}$ & \multirow{3}{*}{$T_{13}$} & Conservative & $I_{31}$ \\
\hline & Steady & $I_{22}$ & & Steady & $I_{21}$ \\
\hline & Radical & $I_{12}$ & & Radical & $I_{11}$ \\
\hline \multirow{3}{*}{$T_{6}$} & Conservative & $I_{5}$ & \multirow{3}{*}{$T_{14}$} & Conservative & $I_{4}$ \\
\hline & Steady & $I_{31}$ & & Steady & $I_{31}$ \\
\hline & Radical & $I_{21}$ & & Radical & $I_{21}$ \\
\hline \multirow{3}{*}{$T_{7}$} & Conservative & $I_{5}$ & \multirow{3}{*}{$T_{15}$} & Conservative & $I_{5}$ \\
\hline & Steady & $I_{32}$ & & Steady & $I_{21}$ \\
\hline & Radical & $I_{22}$ & & Radical & $I_{11}$ \\
\hline \multirow{3}{*}{$T_{8}$} & Conservative & $I_{5}$ & \multirow{3}{*}{$T_{16}$} & Conservative & $I_{5}$ \\
\hline & Steady & $I_{4}$ & & Steady & $I_{4}$ \\
\hline & Radical & $I_{31}$ & & Radical & $I_{21}$ \\
\hline
\end{tabular}

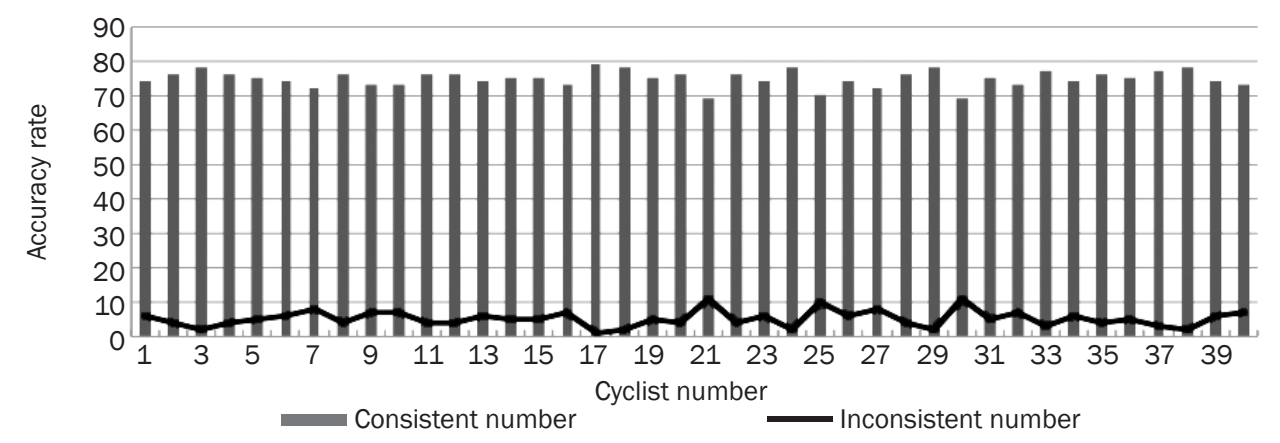

Figure 6 - Comparison between model identification and video observation

environment at different times is revealed in Figures 7-9. The displacement of all kinds of cyclists is described in Figures 10-12.

It can be seen from Figures 7-12 that whether macro-regularity or micro-regularity of the bicycle traffic is taken as valuation index of cyclists' intention identification models, the result of the simulation program operation matches the observed result. It can be also found that the lateral displacement of the radical cyclist is larger than the one of the steady cyclist, and the steady cyclist features a larger lateral displacement than the conservative cyclist.
Above all, the research results show that the simulation has a good consistency with the actual case. Thus, the cyclists' intention is identified by the intention identification model. In order to simplify the research, there still exist some shortcomings:

There are some constraints of time and other objective factors in the process of establishing the intention identification model. These objective factors include traffic investigators, the precision of equipment and other objective conditions. Since the input variables of the identification model are theoretically deduced from the actual data, there are some errors between 


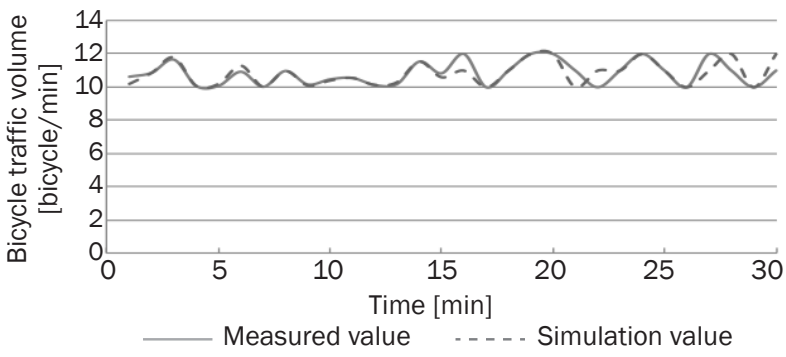

Figure 7 - Simulation verification for traffic flow

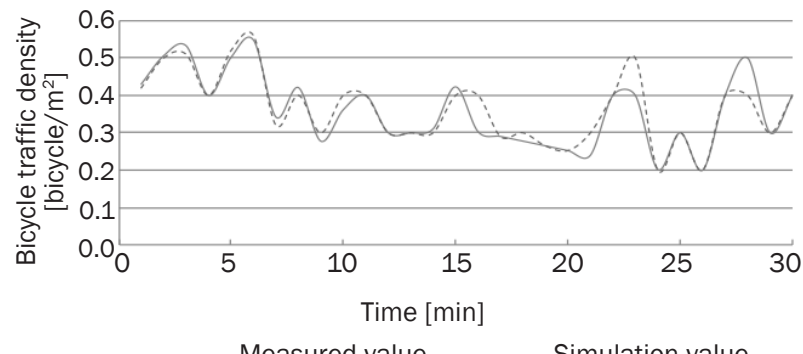

Figure 8 - Simulation verification for traffic density

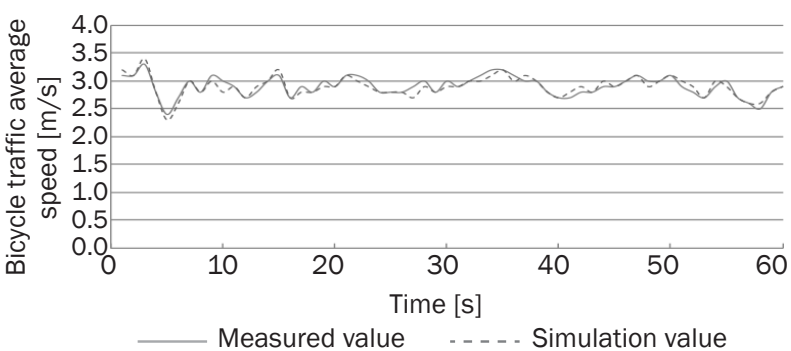

Figure 9 - Simulation verification for average speed

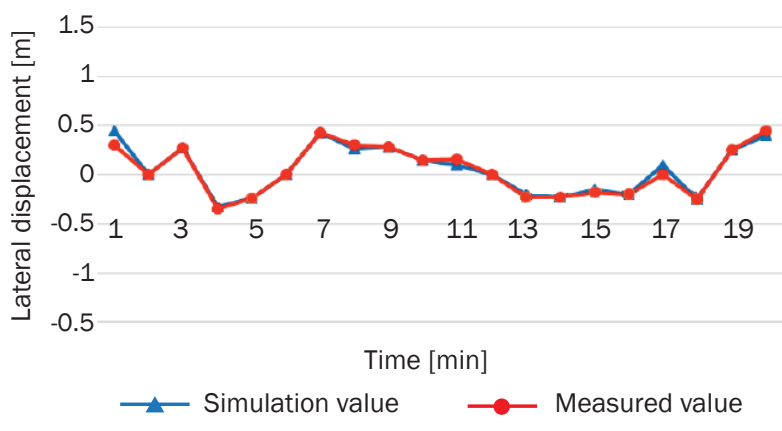

Figure 10 - Radical cyclist's displacement

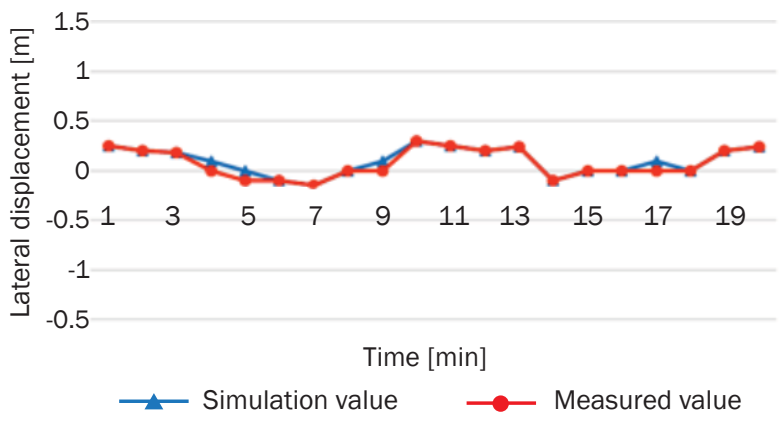

Figure 11 - Steady cyclist's displacement

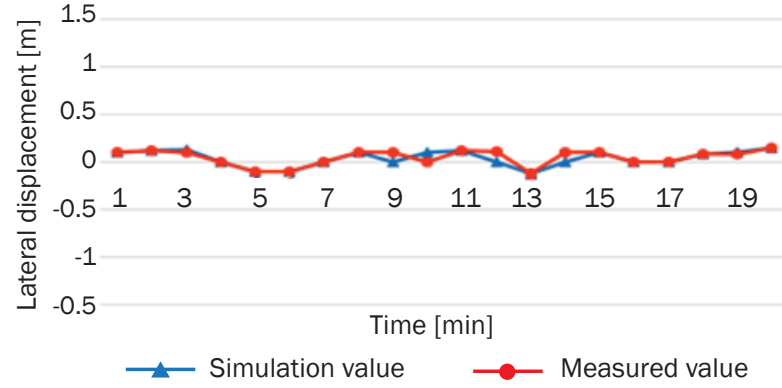

Figure 12 - Steady cyclist's displacement

the cyclists' intention identification result and the actual data. Therefore, the accuracy of the cyclists' intention identification results will be enhanced through improving an experimental method and expanding the experimental sample size in the future studies.

\section{CONCLUSION}

The cyclist's intention is the brain's comprehensive reflection of the riding behaviour. The cognition and disposal of information in the complex pedestrian-bicycle mixed environment are determined by the cyclist's intention. The traffic phase of the pedestrian-bicycle mixed environment is defined and simplified based on the field coupling theory. The characteristic parameters of the cyclists' intention are extracted by adaptive genetic algorithm based on the information entropy. The D-S evidence theory is adopted to establish the cyclists' intention identification model in pedestrian-bicycle mixed sections, and the model is experimentally verified. The results show that the established model realizes the dynamic identification of the cyclists' intention on pedestrian-bicycle mixed sections and provides a guarantee for the study of pedestrian-bicycle coordination, real-time warning, and the improvement of pedestrian-bicycle active safety. It should be pointed out that the cyclist's intention is different in pedestrian-bicycle mixed traffic environment. In order to improve the accuracy of the cyclist's intention identification, the model parameters need to be calibrated according to the specific cyclist and the corresponding pedestrian-bicycle mixed environment.

\section{ACKNOWLEDGEMENTS}

This study is supported by the State Key Laboratory of Automotive Safety and Energy under Project No. KF16232, the National Natural Science Foundation of China (Grant No. 61074140, 61573009, 51508315, 51608313), Natural Science Foundation of Shandong Province (Grant No. ZR2014FM027), Social Science Planning Project of Shandong Province (Grant No. 14CGLJ27), Project of Shandong Province Higher Educational Science and Technology Program (Grant 
No. J15LB07), and Postgraduate Research \& Practice Innovation Program of Jiangsu Province (Grant No. KYCX19_0196).

汪海波 ${ }^{1}$, 博士

电子邮箱: sdlgwanghaibo@sohu.com

司海青1 (通讯作者)，教授，博士

电子邮箱: sihaiqing@126.com

王晓原 ${ }^{2}$, 教授, 博士

电子邮箱: sql370@163.com

1 南京航空航天大学 民航学院/飞行学院, 南京市,

江苏省，中国

2 青岛科技大学 机电工程学院, 青岛市, 山东省, 中国

基于相场耦合的人一自混杂路段骑行者意图辨识

\section{摘要}

自行车是人-自混行路段影响交通安全和通行能力的 主要因素。研究物联网 (人自协同) 条件下人-自混杂环境 骑行者意图的动态辨识, 对适时安全预警、增进人车主动 安全具有重要意义。基于此，本文借鉴相场耦合理论，以 复杂人-自交通相态简化情况为切入点, 综合分析交通相 态的生成、涌现、增长、转移、消退等规律并研究其约简 方法。运用信息熵自适应遗传算法，提取简约交通相态中 不同类型骑行者意图辨识的特征向量，在此基础上进一步 利用证据理论建立自行车骑行意图辨识模型。实验验证表 明, 本文研究的骑行意图特征向量提取方法和辨识模型科 学合理, 可以为将来研究构建人自交互安全系统提供理论 基础。

\section{关键词}

相场耦合; 人-自混行；意图辨识；安全预警；交通相态

\section{REFERENCES}

[1] Hossain M. Capacity estimation of traffic circles under mixed traffic conditions using micro-simulation technique. Transportation Research Part A. 1999;33(1): 47-61.

[2] Zhao D, Wang W, Li C, Li Z, Fu P, Hu X. Modeling of Passing Events in Mixed Bicycle Traffic with Cellular Automata. Transportation Research Record Journal of the Transportation Research Board. 2013;2387(2387): 26-34.

[3] Wang $\mathrm{H}$. New vector field microcosmic model for flow. China Journal of Highway and Transport. 2003;16(2): 99-102.

[4] Jia B, Li X, Jiang R, Gao ZY. Multi-value cellular automata model for mixed bicycle flow. European Physics Journal B. 2007;56(3): 247-252.
[5] Zhao X, Jia B, Gao Z, Jiang R. Traffic interactions between motorized vehicles and nonmotorized vehicles near a bus stop. Journal of Transportation Engineering-ASCE. 2009;135(11): 894-906.

[6] Huang L, Wu J. A Study on the cyclist's behavior at signalized intersections. IEEE Transactions on Intelligent Transportation System. 2004;5(4): 317-322.

[7] Liang X, Mao B, Xu Q. Perceptual Process for Bicyclist Microcosmic Behavior. Procedia - Social and Behavioral Sciences. 2012;43(4): 540-549.

[8] Chen DF. Modeling and Analyzing of Mixed Traffic Flow on the Urban Road Section Based on Social Force. MS thesis. Central South University, China; 2011.

[9] Chen J, Xie Z. Cycle traffic conflict model on urban pedestrian-bicycle paths. Journal of Jilin University (Engineering and Technology Edition). 2009;39(2): 121-125.

[10] Deng J. Mixed Traffic Flow Cellular Automaton Model Based on Shared-Use Sidewalk. Journal of Transportation Systems Engineering and Information Technology. 2011;11(2): 155-159.

[11] Yu Z, Liu P, Long Y. Research Progress of Phase-field Method Based on Ginzburg-Landau Theory. Material \& Heat Treatment. 2008;37(16): 94-98.

[12] Wang X, Zhang J, Ban XJ, Tan D. Dynamic Feature Extraction Method of Driver's Propensity under Complicated Vehicle Group. Advances in Mechanical Engineering. 2013; 1-10.

[13] Wei L, Ying T. Modeling and simulation on bicycle traffic flow based on cellular automaton. Journal of Jilin University (Engineering and Technology Edition). 2011;41(1): 51-55.

[14] Wu L, Wang $X$, Yang $X$, Wang $X$. Study on the recognition of traffic situation and its state transition mechanism. Communications Standardization. 2007;(2-3): 61-65.

[15] Wang X, Zhang J, Liu Y, Wang Y, Wang F, Wang J. The drivers' lane selection model based on mixed fuzzy many-person multi-objective non-cooperative game. Journal of Intelligent \& Fuzzy Systems. 2017;32: 4235-424.

[16] Wang K. Study on the coupling mechanism of vehicle cluster situation and driver's propensity. MS thesis. Shandong University Technology, China; 2015.

[17] Li F, Qian Y, Wang J, Liang J. Multigranulation information fusion: a Dempster-Shafer evidence theory-based clustering ensemble method. Information Sciences. 2016;1(10): 58-63.

[18] Karami AH, Hasanzadeh M. An adaptive genetic algorithm for robot motion planning in 2D complex environments. Computers \& Electrical Engineering. 2015;43(4): 317-329. 